\title{
Role of nutritional status and intervention in oesophageal cancer treated with definitive chemoradiotherapy: outcomes from SCOPE1
}

\begin{abstract}
S Cox ${ }^{1,5}$, C Powell ${ }^{1,5}$, B Carter ${ }^{2,5}$, C Hurt ${ }^{3}$, Somnath Mukherjee ${ }^{\star, 4,6}$ and Thomas David Lewis Crosby ${ }^{1,6}$
${ }^{1}$ Department of Oncology, Velindre Cancer Centre, Cardiff CF14 2TL, UK; ${ }^{2}$ Institute of Primary Care and Public Health, Cardiff University School of Medicine, Neuadd Meirionnydd, Heath Park, Cardiff CF14 4YS, UK; ${ }^{3}$ Wales Cancer Trials Unit, Cardiff University, Neuadd Meirionnydd, Heath Park, Cardiff CF14 4YS, UK and ${ }^{4}$ Department of Oncology, Oxford Cancer Centre, University of Oxford, Level 2 Admin, Churchill Hospital, Oxford OX3 7LE, UK
\end{abstract}

Background: Malnutrition is common in oesophageal cancer. We aimed to identify nutritional prognostic factors and survival outcomes associated with nutritional intervention in the SCOPE1 (Study of Chemoradiotherapy in OesoPhageal Cancer with or without Erbitux) trial.

Methods: Two hundred and fifty eight patients were randomly allocated to definitive chemoradiotherapy (dCRT) $+/-$ cetuximab. Nutritional Risk Index (NRI) scores were calculated; NRI $<100$ identified patients at risk of malnutrition. Nutritional intervention included dietary advice, oral supplementation or major intervention (enteral feeding/tube placement). Univariable and multivariable analyses using Cox proportional hazard modelling were conducted.

Results: At baseline NRI $<100$ strongly predicted for reduced overall survival (hazard ratio (HR) 12.45, 95\% Cl 5.24-29.57; $P<0.001$ ). Nutritional intervention improved survival if provided at baseline (dietary advice (HR $0.12, P=0.004$ ), oral supplementation ( $H R \quad 0.13, P<0.001$ ) or major intervention ( $H R 0.13, P=0.003)$ ), but not if provided later in the treatment course. Cetuximab patients receiving major nutritional intervention had worse outcomes compared with controls (13 vs 28 months, $P=0.003$ ).

Conclusions: Pre-treatment assessment and correction of malnutrition may improve survival outcomes in oesophageal cancer patients treated with dCRT. Nutritional Risk Index is a simple and objective screening tool to identify patients at risk of malnutrition.

Oesophageal cancer is the eighth most common cancer worldwide with a 5-year survival rate of $<20 \%$ (Cancer Research UK, 2016). Malnutrition affects up to $80 \%$ of the patients and is multifactorial in aetiology (Riccardi and Allen, 1991). Patients often present late with obstructive symptoms, cachexia, weight loss due to locally advanced disease. The psychological impact of diagnosis can result in low mood and depression, which may further reduce appetite (Van Cutsem and Arends, 2005).
Definitive chemoradiotherapy (dCRT) is a treatment option for localised oesophageal cancer, particularly in squamous cell carcinoma or in patients with adenocarcinoma deemed unsuitable for surgery (Herskovic et al, 1992; Smith et al, 1998; Cooper et al, 1999; Minsky et al, 2002; Bedenne et al, 2007; Crosby et al, 2013). Nearly half of the patients experience grade 3-4 gastrointestinal toxicities during dCRT (Crosby et al, 2013) and 20\% may require invasive nutritional support (Gwynne et al, 2011). Nutritional

${ }^{\star}$ Correspondence: Dr Somnath Mukherjee; E-mail: somnath.mukherjee@oncology.ox.ac.uk

${ }^{5}$ These authors share co-first authorship.

${ }^{6}$ These authors share co-last authorship.

Received 16 March 2016; accepted 18 April 2016; published online 21 June 2016

(c) 2016 Cancer Research UK. All rights reserved 0007-0920/16

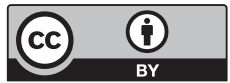


intervention improves weight gain, performance status, tolerability of treatment, overall survival (OS) and quality of life in oncology patients (Lee et al, 2016). However prospective studies specifically evaluating the impact of malnutrition and nutritional intervention in patients with oesophageal cancer treated with dCRT are lacking.

The SCOPE1 (Study of Chemoradiotherapy in OesoPhageal Cancer with or without Erbitux) trial was a randomised controlled phase II/III trial comparing cisplatin-capecitabine-based dCRT for oesophageal cancer with or without cetuximab (Crosby et al, 2013). Two hundred and fifty eight patients were recruited from 36 centres in the UK between February 2008 and January 2012; the trial was stopped at the phase II stage because it met criteria for futility. The study reported an OS detriment in the cetuximab arm (22.1 months (95\% CI 15.1-24.5) vs 25.4 months (95\% CI 20.5-37.9); adjusted HR 1.53 (95\% CI 1.03-2.27); $P=0.035$ ).

The aim of this study was to identify nutritional prognostic factors and the survival outcome of nutritional interventions in patients recruited to SCOPE1. We hypothesised that poor nutritional status at baseline would be associated with worse outcomes and nutritional intervention may improve survival.

\section{MATERIALS AND METHODS}

Study design. Patients were randomised in a $1: 1$ allocation ratio. The control arm received four cycles of chemotherapy with cisplatin $\left(60 \mathrm{mg} \mathrm{m}^{-2}\right.$ IV day 1 of 21$)$ and capecitabine (625 $\mathrm{mg} \mathrm{m}^{-2}$ po bd, continuously). Concurrent conformal radiotherapy ( 50 Gy in 25 fractions over 5 weeks, 2 Gy per fraction) started with cycle 3 (week 7). In the research arm, cetuximab was commenced with cycle $1\left(400 \mathrm{mg} \mathrm{m}^{-2}\right.$ day 1 of week 1 , then $250 \mathrm{mg} \mathrm{m}^{-2}$ weekly thereafter for 11 weeks).

The full trial protocol has been published elsewhere and was approved by the UK Medicines and Healthcare Products Regulatory Agency and a multicentre research ethics committee (Hurt et al, 2011). The SCOPE1 trial was an International Standard Randomised Controlled Trial, number 47718479. Written informed consent was obtained from all recruited patients.

Data collection. Data on the following nutritional parameters were prospectively collected at two time points, baseline and prior to dCRT (week 7): albumin ( $\mathrm{gl}^{-1}$ ), body mass index (BMI, weight $(\mathrm{kg})$ per height ${ }^{2}$ (metres)), Mellow score for dysphagia (grade 0-4) (Mellow and Pinkas, 1985), performance status (0-1) and nutritional intervention received (none, dietary advice, oral supplements or major intervention). Major intervention was defined as enteral feeding via nasogastric/nasojejunal tube placement, percutaneous endoscopic gastrostomy (PEG) or jejunostomy.

Due to difficulties in ascertaining usual body weight in cancer patients, the Lorentz formula was used to calculate ideal body weight (Bouillanne et al, 2005). Percentage weight loss was defined as ((current body weight - ideal body weight)/ideal body weight $) \times 100$. The nutritional risk index $(\mathrm{NRI})$ was calculated at each time point using the following formula: $\mathrm{NRI}=(1.519 \times$ albumin $\left.\mathrm{g} \mathrm{dl}^{-1}\right)+41.7$ (present weight/ideal weight) (Buzby et al, 1988; The Veterans Affairs Total Parenteral Nutrition Cooperative Study Group, 1991; Aziz et al, 2011). Patients were stratified according to the risk of malnutrition: NRI score $\geqslant 100$ : no risk; NRI 97.5-100: mild risk; NRI 83.5-97.5: moderate risk; NRI < 83.5: major risk.

Cross tabulations of median survival were generated for all effect modifiers and compared independently with the NRI and study design stratification variables (treatment as allocated, centre, type of tumour, stage, reason for non-surgical therapy, age, gender, baseline weight and dysphagia score).
Statistical analysis. The main analysis determined the effect modifiers of survival using a multivariable model at baseline. Cox proportion hazards regression was used to model survival. As trial participants were recruited from 36 centres, treatment centre was included as a frailty to adjust for clustering.

The baseline Cox proportion hazards model included variables consistent with the main trial analysis as a priori prognostic variables (centre, disease stage, reason for no surgery, tumour type (adenocarcinoma vs squamous histology), tumour stage, trial arm, performance status, sex, tumour length, radiation dose, cisplatin dose, capecitabine dose and age group). Additional justified effect modifiers (including biochemistry and nutritional parameters) were tested for inclusion in the base model. The main effects of these modifiers and the pre-specified interactions were sequentially introduced in order of statistical importance using a likelihood ratio test $(P \leqslant 0.01)$ independently for each time point (baseline and pre-dCRT). Parameter estimates, standard errors and $P$ values were calculated. The proportional hazards assumption was assessed visually using Kaplan-Meier plots. Non-parametric log-rank tests were used to assess differences in hazard functions across subgroups.

\section{RESULTS}

Data from 258 patients recruited to the SCOPE1 trial were evaluated; details of the main analysis are published elsewhere (Crosby et al, 2013). The median length of follow-up was 25.0 (IQR 12.6-42.7) months. The number of patients in each NRI group at baseline was balanced between the two treatment arms (dCRT alone $v s$ dCRT plus cetuximab, data not shown).

The majority of patients were classified not at risk of malnutrition ( $\mathrm{NRI} \geqslant 100)$ at baseline (217 (84\%) patients); 14 (5\%) were at mild risk (NRI 97.5-100), 22 (9\%) at moderate risk (NRI 83.5-97.5) and only $5(2 \%)$ were calculated to be at major risk (NRI < 83.5). However, after 6 weeks of induction chemotherapy the number of patients at moderate/major malnutrition risk had increased (179 patients (70\%), no risk; 16 (6\%), mild risk; 48 (19\%), moderate risk; 11 (4\%), major risk).

The number of patients receiving nutritional intervention increased during induction chemotherapy (143 (56\%) patients at baseline vs $192(75 \%)$ patients prior to dCRT). Although the number of patients receiving dietary advice alone remained approximately stable (44 (17\%) vs 40 (16\%) patients), the use of oral supplements $(74(29 \%)$ vs $110(43 \%)$ patients) and feeding tubes increased on treatment (25 (10\%) vs 42 (16\%) patients).

Median OS for patients according to NRI score and nutritional intervention received at baseline and prior to dCRT are shown in Tables 1 and 2. Tumour length $(6-8 \mathrm{~cm})$, stage III disease and

Table 1. Median survival time (months) according to maximum nutritional intervention received and nutritional risk index (NRI) score at baseline

\begin{tabular}{|l|c|c|c|c|}
\cline { 2 - 5 } \multicolumn{1}{c|}{} & \multicolumn{4}{c|}{ NRI score } \\
\cline { 2 - 5 } & $\begin{array}{c}\geqslant 100 \\
(\mathbf{N})\end{array}$ & $\begin{array}{c}>\mathbf{9 7 . 5} \\
\mathbf{( N )}\end{array}$ & $\begin{array}{c}<\mathbf{9 7 . 5} \\
(\mathbf{N})\end{array}$ & $\begin{array}{c}\text { All patients } \\
(\mathbf{N})^{\mathbf{a}}\end{array}$ \\
\hline None & $34.5(105)$ & $7.4(3)$ & $3.5(6)$ & $29.2(114)$ \\
\hline Dietary advice & $31.6(39)$ & $28.8(2)$ & $18.4(3)$ & $28.9(44)$ \\
\hline Oral supplements & $24.6(58)$ & $23.9(5)$ & $19.1(11)$ & $23.4(74)$ \\
\hline Major nutritional intervention & $24.7(15)$ & $44.0(4)$ & $10.6(6)$ & $23.6(25)$ \\
\hline All patients & $27.9(217)$ & $21.1(14)$ & $11.3(21)$ & $24.9(257)$ \\
\hline a Data on nutritional intervention received at baseline were missing for one patient. \\
\hline
\end{tabular}


Table 2. Median survival time (months) according to maximum nutritional intervention received and nutritional risk index (NRI) score prior to definitive chemoradiotherapy (dCRT)

\begin{tabular}{|c|c|c|c|c|}
\hline & \multicolumn{3}{|c|}{ NRI score } & \multirow{2}{*}{$\begin{array}{c}\text { All patients } \\
(\mathrm{N})^{\mathrm{a}}\end{array}$} \\
\hline & $\begin{array}{c}\geqslant 100 \\
(N)\end{array}$ & $\begin{array}{c}>97.5 \\
(N)\end{array}$ & $\begin{array}{c}<97.5 \\
(N)\end{array}$ & \\
\hline None & $35.7(56)$ & $23.1(9)$ & $11.3(1)$ & $35.1(66)$ \\
\hline Dietary advice & $36.7(29)$ & $12.2(10)$ & & 35.9 (39) \\
\hline Oral supplements & $24.8(74)$ & $19.1(29)$ & $11.3(6)$ & $23.2(109)$ \\
\hline Major nutritional intervention & $24.7(20)$ & $16.5(16)$ & $13.0(4)$ & $21.4(40)$ \\
\hline All patients & 30.2 (179) & $18.3(64)$ & $12.5(11)$ & $24.9(254)$ \\
\hline
\end{tabular}

receiving $<50 \mathrm{~Gy}$ radiotherapy were identified as independent prognostic factors in multivariable analysis $(P<0.01)$, (Table 3 ).

Overall survival was significantly worse in patients classified to be at the risk of malnutrition at baseline $(\mathrm{NRI}<100$, median survival time 15.7 months; IQR 7.4-25.8) compared with patients not at risk (NRI $\geqslant 100$, median survival time 31.6 months; IQR 14.7-58.0) (HR 12.5 (95\% CI 5.2-29.6), $P<0.001$ ) (Figure 1). In these at-risk patients, providing nutritional intervention at baseline was associated with an improved OS (dietary advice (HR 0.12 (0.03-0.51), $P=0.004$ ), oral supplements (HR 0.13, (0.04-0.39), $P<0.001)$ and major intervention (HR $0.13(0.03-0.50), P=0.003$ ) (Table 3; Figure 2). Univariable main effects not included in the multivariable model are shown in Supplementary Table S1.

The median survival for patients with NRI $<100$ and NRI $\geqslant 100$ prior to starting dCRT was 15.4 and 30.3 months, respectively, but after adjustment for other factors this was not significant in the multivariable model (HR 1.79 (0.64-5.04), $P=0.27$, full model not shown). Furthermore, nutritional intervention provided to at-risk patients after the commencement of induction chemotherapy was no longer associated with a survival benefit (dietary advice alone HR $1.31, P=0.72$; oral supplements $\mathrm{HR} 0.86, P=0.81$; major intervention HR 0.84, $P=0.81$ ).

The only survival difference based on nutritional parameters between the trial arms was seen in patients who required major nutritional intervention; those allocated to dCRT plus cetuximab had a shorter OS than those treated with dCRT alone at both baseline (HR 4.7 (1.4-15.70), $P=0.01$ ) and pre-dCRT (HR 5.4 (1.75-16.36), $P=0.003)$.

\section{DISCUSSION}

This study suggests that increased nutritional risk at baseline is associated with reduced survival in patients with localised oesophageal cancer treated with dCRT. An NRI score $<100$ strongly predicted for reduced OS. An improvement in survival was observed following baseline nutritional intervention with dietary advice, oral supplementation or major intervention. A similar benefit was not observed if nutritional intervention occurred later in the treatment course.

To our knowledge this is the first evaluation of prospectively collected data to demonstrate the benefit of nutritional intervention in oesophageal cancer. Other groups have identified prognostic nutritional factors in patients treated with dCRT. Thomas et al (2004) performed recursive partitioning analysis of pre-treatment variables in 416 patients; only pre-treatment weight loss $>10 \%$ in the 6 months prior to treatment was identified as a significant factor. BMI $>18 \mathrm{~kg} \mathrm{~m}^{-2}$, Atkinson dysphagia score $<2$, dose of RT $>50 \mathrm{~Gy}$ and complete response to CRT were found to be independent prognostic factors for survival in a retrospective analysis of 105 patients (Di Fiore et al, 2007). In a more recent study by the same group, OS was 25 months in patients with persistent malnutrition compared with 42 months in those who remained well nourished during CRT (Di Fiore et al, 2014). There is also evidence for nutritional factors as predictors of benefit following dCRT. Di Fiore et al (2007) found a significant difference in baseline percentage weight loss, albumin level and BMI between responders and non-responders; response rates to dCRT were significantly lower in patients with malnutrition at baseline and during treatment.

Malnutrition in patients treated for oesophageal cancer is common and may be related to the disease or its treatment (Muscaritoli et al, 2011). Appropriate nutritional support is important for maintaining treatment intensity and may influence outcome (Lee et al, 2016). A formalised nutritional pathway for patients receiving dCRT significantly reduced complications with less weight loss, fewer unplanned hospital admissions and greater radiotherapy completion rates observed (Odelli et al, 2005). Concerns regarding the use of PEG feeding tubes in oesophageal cancer relating to safety of dilatation and potential for inoculation metastasis have been raised (Singh and Gelrud, 2015). However in a retrospective analysis, PEG placement was successful in the majority of patients prior to multimodality treatment for oesophageal cancer and was significantly related to attainment of target doses of chemoradiotherapy $(P=0.034)$, and survival at 12 months $(P=0.02)$ (Margolis et al, 2003). In our study, patients with $\mathrm{NRI} \geqslant 100$ at baseline requiring major nutritional intervention had a worse outcome than those who required no nutritional support (24.7 vs 34.5 months, Table 1). This may represent patients with significant dysphagia at presentation due to more locally advanced disease and subsequent reduced survival rates. For patients with mild (NRI 97.5-100) or moderate/major (NRI <97.5) risk, major nutritional intervention improved survival (7.4 vs 44 months and 3.5 vs 10.6 months, respectively, Table 1).

Patients allocated to cetuximab arm who required a major nutritional intervention at baseline had worse survival rates compared with controls (13.3 vs 28 months); a similar finding was observed if nutritional intervention occurred prior to dCRT (13.3 vs 31.1 months). The cause for this survival difference is unclear and based on small patient numbers, however, one hypothesis is that fewer patients receiving cetuximab completed the standard protocol treatment, with significant differences in the number of chemotherapy cycles received and total radiotherapy dose delivered (Crosby et al, 2013).

Our study has limitations; first, only $16 \%$ of the patients were deemed at risk of malnutrition at baseline, which is lower than anticipated. This may represent selection bias as only patients with performance status $0-1$ were recruited, or an inadequacy in dietetic screening as formal dietetic assessment was not a trial prerequisite. Second, although the nutritional data were collected prospectively, the analysis itself is retrospective and requires further prospective validation. Third, although data on whether patients received nutritional intervention were collected, the details of the intervention, intensity of dietetic follow-up or variation between centres were not collected. A randomised control trial of 'conventional hospital protocol nutritional intervention' $v s$ 'NRI-directed nutritional intervention' may be required to assess the true value of NRI-directed intervention in this patient group.

In conclusion, assessment and correction of poor nutritional state at baseline may be a simple and cost-effective intervention that improves survival outcomes in oesophageal cancer patients treated with dCRT. Nutritional Risk Index serves as a simple and objective screening tool to identify patients at risk of malnutrition. In this study, the benefit of nutritional intervention was no longer observed once treatment had commenced, highlighting the need for early nutritional assessment and intervention. 
Table 3. Univariable and multivariable analysis of baseline prognostic factors of overall survival

\section{Survival Univariable analysis}

\begin{tabular}{|c|c|c|c|c|c|}
\hline & $N$, median (Q3-Q1) & $\mathrm{HR}, 95 \% \mathrm{Cl}$ & $P$-value & $\mathrm{HR}, 95 \% \mathrm{Cl}$ & $P$-value \\
\hline \multicolumn{6}{|l|}{ Age } \\
\hline$<70$ years & $160,26.2(43-14.3)$ & \multicolumn{2}{|c|}{ Reference } & \multicolumn{2}{|c|}{ Reference } \\
\hline$\geqslant 70$ years & $98,22.6(40.3-9.2)$ & $1.33,(0.98-1.81)$ & 0.068 & $1.19,(0.82-1.72)$ & 0.366 \\
\hline \multicolumn{6}{|l|}{ Gender } \\
\hline Male & 145,24 (38.6-11.3) & $1.47,(1.07-2.00)$ & 0.016 & $1.38,(0.95-2.02)$ & 0.095 \\
\hline Female & $113,26.1(46.9-14.3)$ & \multicolumn{2}{|c|}{ Reference } & \multicolumn{2}{|c|}{ Reference } \\
\hline \multicolumn{6}{|c|}{ Performance status } \\
\hline 0 & $131,27.2(44-14.7)$ & \multicolumn{2}{|c|}{ Reference } & \multicolumn{2}{|c|}{ Reference } \\
\hline 1 & $127,24.5(40.3-10)$ & $1.17,(0.86-1.59)$ & 0.320 & $0.96,(0.67-1.39)$ & 0.833 \\
\hline \multicolumn{6}{|c|}{ Tumour length } \\
\hline$<2 \mathrm{~cm}$ & $56,30.7(46.4-12.1)$ & \multicolumn{2}{|c|}{ Reference } & \multicolumn{2}{|c|}{ Reference } \\
\hline $2-4 \mathrm{~cm}$ & $85,30.3(46.9-14.8)$ & $1.00,(0.63-1.57)$ & 0.992 & $1.16,(0.70-1.89)$ & 0.568 \\
\hline $4-6 \mathrm{~cm}$ & $55,24.9(42.4-11.5)$ & $1.48,(0.93-2.36)$ & 0.102 & $1.40,(0.79-2.47)$ & 0.250 \\
\hline $6-8 \mathrm{~cm}$ & $62,18.2(35.9-10)$ & $1.87,(1.18-2.96)$ & 0.008 & $1.81,(1.05-3.12)$ & 0.034 \\
\hline
\end{tabular}

Stage

$103,35.9(46.6-15.3)$

I+II

$155,23.2(37-11.3)$

Reference

0.002

Reference

III

Tumour type

Squamous cell

Adenocarcinoma

188, 25.4 (43.3-13.6)

$70,23.2(39.1-10.2)$

$1.66,(1.20-2.30)$

(2)

$\mid$

0.027

Reason for no surgery

Patient choice
Comorbidity

Local extent

97, 26.7 (46.6-14.7)

$36,31.6(42.7-11.1)$

$122,24(40.6-11.5)$

\begin{tabular}{l|l}
\multicolumn{2}{r}{ Reference } \\
$1.28,(0.92-1.78)$ &
\end{tabular}

\begin{tabular}{|l|l|l|r}
\multicolumn{2}{|c|}{ Reference } & \multicolumn{2}{|c}{ Reference } \\
\hline $1.28,(0.92-1.78)$ & 0.144 & $0.97,(0.62-1.52)$ & 0.907
\end{tabular}

Reference

\section{Treatment arm}

dCRT only

dCRT + cetuximab

$129,23.3(39.4-10.2)$

Full radiation protocol dose

Yes

No

$217,30.1(46-14.9)$

$41,8.2(20.8-2.9)$

$1.24,(0.79-1.94)$
$1.20,(0.86-1.69)$

Reference

\begin{tabular}{r|r} 
& Reference \\
\hline 0.350 & $0.93,(0.51-1.70)$
\end{tabular}

\begin{tabular}{l|l}
$1.20,(0.86-1.69)$ & 0.285
\end{tabular}

\begin{tabular}{l|l}
0.350 & $0.93,(0.51-1.70)$ \\
0.285 & $0.87,(0.58-1.29)$
\end{tabular}

0.817

0.478

\section{$\%$ of full cisplatin protocol dose}

\section{$\geqslant 95 \%$}

$\geqslant 75-<95 \%$

$\geqslant 50-<75 \%$

$<50 \%$

106, 35.2 (46.9-16.9)

$76,29(44.3-15.3)$

$41,18.4(36-12.5)$

$35,10.2(24.7-5.9)$

$1.27,(0.94-1.71)$

0.125

$0.82,(0.49-1.37)$

Reference

$\%$ of full capecitabine protocol dose

\begin{tabular}{|c|c|c|c|c|c|}
\hline$\geqslant 95 \%$ & $82,32.8(45.3-14.9)$ & \multicolumn{2}{|c|}{ Reference } & \multicolumn{2}{|c|}{ Reference } \\
\hline$\geqslant 75-<95 \%$ & $90,28.1(45.9-14.8)$ & $1.07,(0.73-1.59)$ & 0.719 & $0.99,(0.62-1.58)$ & 0.956 \\
\hline$\geqslant 50-<75 \%$ & $52,22.5(43.3-7.7)$ & $1.58,(1.03-2.41)$ & 0.035 & $0.97,(0.54-1.73)$ & 0.907 \\
\hline$<50 \%$ & $34,15.6(23.2-5.9)$ & $2.34,(1.46-3.76)$ & $<0.001$ & $0.73,(0.32-1.69)$ & 0.465 \\
\hline
\end{tabular}

\section{NRI}

\section{$\geqslant 100$}

217,28 (45.9-14.2)

$41,15.6(24.5-8)$

$3.46,(2.36-5.07)$

Reference

$<100$

Nutritional intervention

None

Dietary advice alone

Oral supplements

$114,29.2(46.8-12.8)$

Major intervention

$44,29(44.6-15.7)$

74, $23.4(35.8-10.9)$

25, $23.7(40.3-10)$

\begin{tabular}{|l|r|}
\hline \multicolumn{2}{|c|}{ Reference } \\
\hline $1.29,(0.88-1.90)$ & 0.184 \\
$2.20,(1.44-3.38)$ & $<0.001$ \\
$3.17,(2.00,5.03)$ & $<0.001$
\end{tabular}

$<0.001$

$2.92,(1.49-5.75)$

Reference

0.440

Nutritional intervention in the CRT + cetuximab group

None

Dietary advice alone

Oral supplements

Major intervention

\begin{tabular}{l|l}
\multicolumn{2}{c}{ Reference } \\
\hline $2.26,(1.54-3.30)$ & $<0.0$
\end{tabular}

$<0.001$

Reference

12.45, (5.24-29.6) $\quad<0.001$

Nutritional intervention in those with a baseline NRI $<100$

\section{None}

Dietary advice alone

Oral supplements

Major intervention

1.05, (0.67-1.63) $\quad 0.835$

$1.49,(1.04-2.15) \quad 0.030$

1.20, (0.69-2.08)

0.525

Re
$1.11,(0.70-1.75)$
$1.76,(1.00-3.13)$

$1.80,(0.86-3.76)$

Reference

0.002

Abbreviations: $\mathrm{Cl}=$ confidence interval: $\mathrm{CRT}=$ chemoradiotherapy; $\mathrm{dCRT}=$ definitive chemoradiotherapy: $\mathrm{HR}=$ hazard ratio; $\mathrm{NRI}=$ nutritional risk index. 


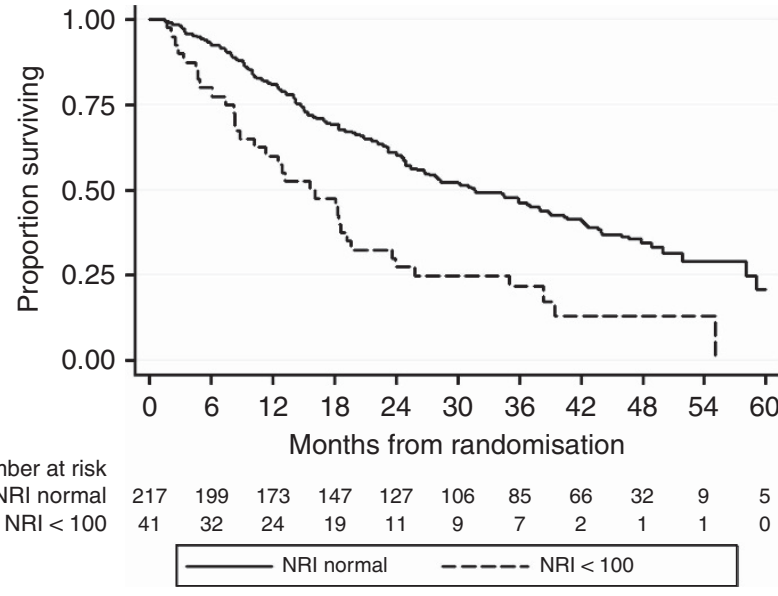

Figure 1. Kaplan-Meier curve of overall survival according to baseline NRI score (log-rank $P$-value $<0.001)$. Abbreviation: NRI, nutritional risk index.

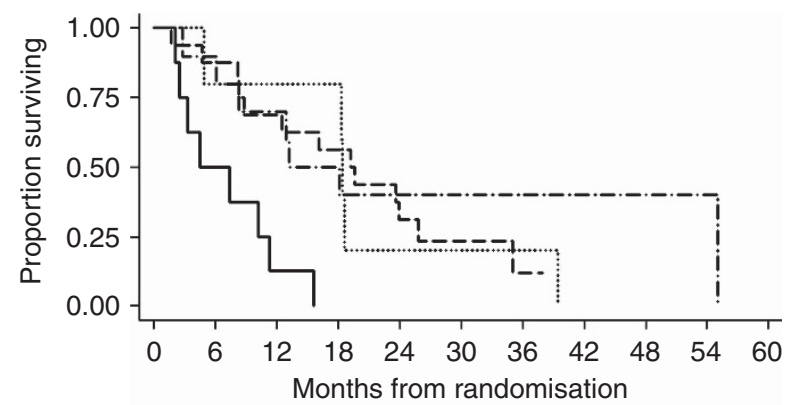

Number at risk No intervention 9 Dietary advice 5
Oral 16 Oral 16

$\begin{array}{cc}4 & 1 \\ 4 & 4 \\ 14 & 11 \\ 9 & 7\end{array}$

$\begin{array}{ll}0 \\ 4 & 1 \\ 9 & 5\end{array}$

$\begin{array}{llllll}0 & 0 & 0 & 0 & 0 & 0 \\ 1 & 1 & 0 & 0 & 0 & 0 \\ 3 & 1 & 0 & 0 & 0 & 0 \\ 4 & 4 & 2 & 1 & 1 & 0\end{array}$

Major intervention 10

$$
\begin{array}{ll}
\text { - No intervention } & \text {................. Dietary advice } \\
\text { - - - Oral intervention } & \text {-._....- Major intervention }
\end{array}
$$

Figure 2. Kaplan-Meier curve of overall survival for patients with baseline $\mathrm{NRI}<100$ according to nutritional intervention received at baseline (log-rank $P=0.001$ ). Abbreviation: NRI, nutritional risk index.

\section{ACKNOWLEDGEMENTS}

SM is partly funded by MRC and supported by NIHR Oxford Biomedical Research Centre. The SCOPE1 trial was supported by Cancer Research UK (grant number C20177/A7256).

\section{CONFLICT OF INTEREST}

Merck Serono supplied free cetuximab, drug labelling and distribution in the SCOPE1 trial but had no role in the design of the study, collection, analysis and interpretation of the data or writing of this report. SC has received educational sponsorship from Merck Serono and Pfizer to attend an international conference. The remaining authors declare no conflict of interest.

\section{REFERENCES}

Aziz EF, Javed F, Pratap B, Musat D, Nader A, Pulimi S, Alivar CL, Herzog E, Kukin ML (2011) Malnutrition as assessed by nutritional risk index is associated with worse outcome in patients admitted with acute decompensated heart failure: an ACAP-HF data analysis. Heart Int 6: e2.

Bedenne L, Micel P, Bouché O, Milan C, Mariette C, Conroy T, Pezet D, Roullet B, Seitz JF, Herr JP, Paillot B, Arveux P, Bonnetain F, Binquet C (2007) Chemoradiation followed by surgery compared with chemoradiation alone in squamous cancer of the Esophagus: FFCD 9102. J Clin Oncol 25: 1160-1168.

Bouillanne O, Morineau G, Dupont C, Coulombel I, Vincent JP, Nicolis I, Benazeth S, Cynober L, Aussel C (2005) Geriatric nutritional risk index: a new index for evaluating at-risk elderly medical patients. Am J Clin Nutr 82: 777-783.

Buzby GP, Knox LS, Crosby LO, Eisenberg JM, Haakenson CM, McNeal GE, Page CP, Peterson OL, Reinhardt GF, Williford WO (1988) Study protocol: a randomized clinical trial of total parenteral nutrition in malnourished surgical patients. Am J Clin Nutr 47: 366-381.

Cancer Research UK (2016) Oesophageal cancer statistics [online]. Available at http://www.cancerresearchuk.org/health-professional/cancer-statistics/ statistics-by-cancer-type/oesophageal-cancer (accessed 26 January 2016).

Cooper JS, Guo MD, Herskovic A, Macdonald JS, Martenson JA JR, Al-Sarraf M, Byhardt R, Russell AH, Beitler JJ, Spenser S, Asbell SO, Graham MW, Leichman LL (1999) Chemoradiotherapy of locally advanced esophageal cancer: long-term follow-up of a prospective randomized trial (RTOG 85-01). Radiation Therapy Oncology Group. JAMA 281: 1623-1627.

Crosby T, Hurt CN, Falk S, Gollins S, Mukherjee S, Staffurth J, Ray R, Bashir N, Bridgewater JA, Geh JI, Cunningham D, Blazeby J, Roy R, Maughan T, Griffiths G (2013) Chemoradiotherapy with or without cetuximab in patients with oesophageal cancer (SCOPE1): a multicentre, phase 2/3 randomised trial. Lancet Oncol 14: 627-637.

Di Fiore F, Lecleire S, Pop D, Rigal O, Hamidou H, Paillot B, Ducrotté P, Lerebours E, Michel P (2007) Baseline nutritional status is predictive of response to treatment and survival in patients treated by definitive chemoradiotherapy for a locally advanced esophageal cancer. Am J Gastroenterol 102: 2557-2563.

Di Fiore A, Lecleire S, Gangloff A, Rigal O, Benyoucef A, Blondin V, Sefrioui D, Quiesse M, Iwanicki-Caron I, Michel P, Di Fiore F (2014) Impact of nutritional parameter variations during definitive chemoradiotherapy in locally advanced oesophageal cancer. Dig Liver Dis 46: 270-275.

Gwynne S, Hurt C, Evans M, Holden C, Vout L, Crosby T (2011) Definitive chemoradiation for oesophageal cancer-a standard of care in patients with non-metastatic oesophageal cancer. Clin Oncol (R Coll Radiol) 23: $182-188$.

Herskovic A, Martz K, al-Sarraf M, Leichman L, Brindle J, Kaitkevicius V, Cooper J, Byhardt R, Davis L, Emami B (1992) Combined chemotherapy and radiotherapy compared with radiotherapy alone in patients with cancer of the esophagus. N Engl J Med 326: 1593-1598.

Hurt CN, Nixon LS, Griffiths GO, Al-Mokhtar R, Gollins S, Staffurth JN, Phillips CJ, Blazeby JM, Crosby TD (2011) SCOPE1: a randomised phase II/III multicentre clinical trial of definitive chemoradiation, with or without cetuximab, in carcinoma of the oesophagus. BMC Cancer 11: 466 .

Lee JL, Leong LP, Lim SL (2016) Nutrition intervention approaches to reduce malnutrition in oncology patients: a systematic review. Support Care Cancer 24: 469-480.

Margolis M, Alexander P, Trachiotis GD, Gharagozloo F, Lipman T (2003) Percutaneous endoscopic gastrostomy before multimodality therapy in patients with esophageal cancer. Ann Thorac Surg 76: 1694-1697.

Mellow MH, Pinkas H (1985) Endoscopic laser therapy for malignancies affecting the esophagus and gastroesophageal junction. Arch Intern Med 145: 1443-1446.

Minsky BD, Pajak TF, Ginsberg RJ, Pisansky TM, Martenson J, Komaki R, Okawara G, Rosenthal SA, Kelsen DP (2002) INT 0123 (Radiation Therapy Oncology Group 94-05) phase III trial of combined-modality therapy for esophageal cancer: high-dose versus standard-dose radiation therapy. J Clin Oncol 20: 1167-1174.

Muscaritoli M, Molfino A, Gioia G, Laviano A, Rossi Fanelli F (2011) The "parallel pathway": a novel nutritional and metabolic approach to cancer patients. Intern Emerg Med 6: 105-112.

Odelli C, Burgess D, Bateman L, Hughes A, Ackland S, Gillies J, Collins CE (2005) Nutrition support improves patient outcomes, treatment tolerance and admission characteristics in oesophageal cancer. Clin Oncol (R Coll Radiol) 17: 639-645. 
Riccardi D, Allen K (1991) Nutritional management of patients with esophageal and esophagogastric junction cancer. Cancer Control 6: 64-72.

Singh A, Gelrud A (2015) Adverse events associated with percutaneous enteral access. Gastrointest Endosc Clin N Am 25: 71-82.

Smith TJ, Ryan LM, Douglass Jr HO, Haller DG, Dayal Y, Kirkwood J, Tormey DC, Schutt AJ, Hinson J, Sischy B (1998) Combined chemoradiotherapy $v s$. radiotherapy alone for early stage squamous cell carcinoma of the eosphagus: a study of the Eastern Cooperative Oncology Group. Int J Radiat Oncol Biol Phys 42: 269-276.

The Veterans Affairs Total Parenteral Nutrition Cooperative Study Group (1991) Perioperative total parenteral nutrition in surgical patients. N Engl J Med 325: 525-532.
Thomas Jr CR, Berkey BA, Minsky BD, Gaspar LE, Herskovic A, Rich TA, Gunderson LL (2004) Recursive partitioning analysis of pretreatment variables of 416 patients with locoregional esophageal cancer treated with definitive concomitant chemoradiotherapy on Intergroup and Radiation Therapy Oncology Group trials. Int J Radiat Oncol Biol Phys 58: 1405-1410.

Van Cutsem E, Arends J (2005) The causes and consequences of cancerassociated malnutrition. Eur J Oncol Nurs 9: 51-63.

(c) (i) This work is licensed under the Creative Commons Attribution 4.0 International License. To view a copy of this license, visit http://creativecommons.org/licenses/by/4.0/

Supplementary Information accompanies this paper on British Journal of Cancer website (http://www.nature.com/bjc) 